\title{
Relationships between nanostructure and dynamic-mechanical properties of epoxy network containing PMMA-modified silsesquioxane
}

\author{
S. Zaioncz ${ }^{1}$, K. Dahmouche ${ }^{2}$, C. M. Paranhos ${ }^{1,2}$, R. A. S. San Gil ${ }^{3}$, B. G. Soares ${ }^{1 *}$ \\ ${ }^{1}$ Instituto de Macromoléculas, Universidade Federal do Rio de Janeiro Centro de Tecnologia, Bloco J, Ilha do Fundão, \\ 21945-970, Rio de Janeiro, P.O.Box 68525, RJ, Brazil \\ ${ }^{2}$ Universidade Estadual da Zona Oeste, Rio de Janeiro, RJ, Brazil \\ ${ }^{3}$ Instituto de Quimica, Universidade Federal do Rio de Janeiro, RJ, Brazil
}

Received 24 January 2009; accepted in revised form 28 March 2009

\begin{abstract}
A new class of organic-inorganic hybrid nanocomposites was obtained by blending PMMA-modified silsesquioxane hybrid materials with epoxy matrix followed by curing with methyl tetrahydrophthalic anhydride. The hybrid materials were obtained by sol-gel method through the hydrolysis and polycondensation of the silicon species of the hybrid precursor, 3-methacryloxypropyltrimethoxysilane (MPTS), simultaneously to the polymerization of the methacrylate (MMA) groups covalently bonded to the silicon atoms. The nanostructure of these materials was investigated by small angle X-ray scattering (SAXS) and correlated to their dynamic mechanical properties. The SAXS results revealed a hierarchical nanostructure consisting on two structural levels. The first level is related to the siloxane nanoparticles spatially correlated in the epoxy matrix, forming larger hybrid secondary aggregates. The dispersion of siloxane nanoparticles in epoxy matrix was favored by increasing the MMA content in the hybrid material. The presence of small amount of hybrid material affected significantly the dynamic mechanical properties of the epoxy networks.
\end{abstract}

Keywords: nanocomposites, epoxy, SAXS, sol-gel, dynamic-mechanical properties

\section{Introduction}

Epoxy resins are widely used thermosets in several industrial applications because of their high mechanical and adhesion characteristics associated to the good chemical resistance. However their brittleness limits some important applications mainly as high performance adhesives and composites. The incorporation of liquid rubber components constitutes a usual approach for improving toughness of these materials, but with frequent detriment of stiffness and modulus [1-6]. The increased demand for higher performance materials which combine improved strength, hardness, toughness and heat resistance has motivated several investiga- tions on the field of organic/inorganic nano-hybrid materials. The nano-sized inorganic fillers incorporated into an epoxy matrix are expected to provide a synergistic combination of individual organic/inorganic component properties, thanks to the dramatic increase of the interface area between the components [7-8]. Moreover, the dispersion of the filler in nano-size dimensions may also provide a homogeneous morphology throughout the composite, which greatly improves the reproducibility of its properties [9-10].

Among several inorganic nano-particles that can be incorporated into the epoxy resin, those constituted by polyhedral oligomeric silsesquioxane (POSS)

*Corresponding author, e-mail: bluma@ima.ufrj.br

(C) BME-PT 
have been emerging as a new and versatile technology because of the simplicity of their synthesis and processing. Moreover they offer a unique opportunity for preparing truly dispersed nanocomposites [10-12]. POSS is a class of important nanosized cage-like compounds, obtained by a sol-gel process involving hydrolysis and condensation of trialkoxy silanes bearing an organic group [13-16]. Depending on the reaction parameters it is possible to obtain molecules with different structures. Additionally, the POSS structure may be completely condensed, presenting a cube octameric framework constituted by an inorganic core formed by silica and surrounding by eight organic corner groups or be partially condensed, presenting some hydroxyl groups $[17,18]$. The nature of the $R$ groups is very important to provide good interaction between the filler and the epoxy matrix, which in turn affects the degree of dispersion, the local structure and mobility of the polymer chains. Consequently an improvement of thermal, oxidative and dimensional stability as well as mechanical performance of many polymeric resins is usually achieved. Mono- and multi-functionalized silsesquioxanes containing epoxy [7, 19-27], amino [28-32], mercapto [33], maleimido [34, 35], and isocyanate groups [36] have been successfully employed in the preparation of epoxy resin-based hybrid materials. Mono-functionalized silsesquioxanes are prepared by corner capping reaction of a trisilanol with a functionalized trialkoxy silane, and results in POSS molecules grafted onto the epoxy matrix [14]. Polyfunctional POSS is obtained from the hydrolysis/ condensation of a functionalized trialkoxi silane resulting in polymer networks with the POSS cage acting as a crosslinking site [7]. Epoxy resin/POSS hybrid materials are prepared by mixing both components [23] or by performing the hydrolysis/condensation of the silane precursor in the presence of the epoxy matrix. When the POSS molecule is prepared in situ, the inorganic siloxane particles are more linked with the epoxy network because the alkoxy groups of the silane precursor can react with the hydroxyl groups formed by the ring opening reaction of the epoxy groups, through an ether exchange reaction [20].

Inorganic-organic hybrid compounds constituted by siloxane particles chemically linked to another polymer that is miscible with the epoxy matrix is also a good strategy for the development of new nanostructured epoxy hybrid networks. In this sense, an interesting family that fulfills this requirement is that obtained from the copolymerization between a vinyl-functionalized trialkoxysilane precursor and methyl methacrylate (MMA). Hybrid compounds have been prepared by different methodologies to develop materials with adjustable transparency, refractive index and hardness [37-40]. However, to the best of our knowledge, there are no reports in the accessible literature, regarding its use as epoxy modifier.

The present work aims for the first time to study the effect of Silsesquioxane-PMMA hybrid materials (SSO-PMMA) on the nanostructural features of the epoxy networks. The hybrid materials were synthesized by the hydrolysis/condensation of the (3methacryloxypropyl) trimethoxy silane (MPTS) precursor simultaneously to its free radical copolymerization with methyl methacrylate. This process gives rise to a crosslinked hybrid compound with the PMMA chains surrounding the inorganic siloxane particles [41-43]. This structural feature contributes for a better filler-epoxy matrix interaction since the PMMA chains chemically linked to the silsesquioxane moiety can physically interact with the epoxy matrix, providing a good anchorage between the components. Moreover, when the MMA is employed as the co-monomer, their chains help to keep the inorganic particle away from each other, decreasing the chance of filler aggregation. The nanoscopic features of the epoxy network modified with these hybrid materials were determined by small angle X-ray scattering (SAXS) and were related to the dynamic mechanical properties.

\section{Experimental}

\subsection{Materials}

3-methacryloxypropyltrimethoxysilane (MPTS) was purchased from Fluka and used without further purification. The monomers methyl methacrylate (MMA) was obtained from Methacryl do Brasil S.A. and distilled under reduced pressure. Benzoyl peroxide (BPO), supplied by Vetec Ind. Quim. Ltda (Rio de Janeiro, Brazil) was used as the free radical initiator, and was recrystallized from chloroform/ ether. The epoxy resin used in this study was diglycidyl ether of bisphenol-A (DGEBA), DER 331 (Dow Chemical) and presents the following characteristics: $\left\langle M_{n}\right\rangle=384$; viscosity $=12,000 \mathrm{mPa} \cdot \mathrm{s}$; 
epoxide equivalent $=192 \mathrm{~g} \cdot \mathrm{eq}^{-1}$. Methyl tetrahydro phthalic anhydride (Aradur HY 2123) used as the curing agent was supplied by Hunstman (São Paulo, Brazil). This agent contains $2 \%$ in weight of 2-ethyl-4-methyl-imidazole, used as catalyst of the curing process.

\subsection{Synthesis of Silsesquioxane-PMMA hybrid materials}

$0.4 \mathrm{~g}(1.68 \mathrm{mmol})$ of MPTS was mixed with $0.2 \mathrm{ml}$ of ethanol containing $0.10 \mathrm{ml}$ of concentrated hydrochloric acid. This proportion corresponds to a $\mathrm{H}_{2} \mathrm{O} / \mathrm{MPTS}$ molar ratio of 3:1 and a $\mathrm{pH}$ of solution of 1.0. Then, desired amounts of MMA and BPO were added into the flask. (The amount of BPO related to the vinyl monomer content was $3 \mathrm{~mol} \%$ ). The reaction medium was stirred at $45^{\circ} \mathrm{C}$ for $144 \mathrm{~h}$, until gelation. The ethanol and $\mathrm{H}_{2} \mathrm{O}$ used as solvents, the methanol produced from the silane precursor condensation, as well as the remaining non reacted MMA monomers were removed under vacuum, and the resulting monolithic dried gel was milled and stored under nitrogen. Table 1 presents the Silsesquioxane-PMMA compositions obtained theoretically from the initial formulations. The values are close to those obtained experimentally from TGA measurements, which were also presented in the same table.

\subsection{Preparation of epoxy-based nanocomposites}

6 parts of Silsesquioxane-PMMA was dispersed into 100 parts of anhydride used as the hardener, at $50^{\circ} \mathrm{C}$ under intensive mixing. Then, 100 parts of DGEBA was added. The mixture was stirred, degassed, poured into an open silicon mold and cured at $80^{\circ} \mathrm{C}$ for $3 \mathrm{~h}$, followed by a post curing treatment at $110^{\circ} \mathrm{C}$ for $3 \mathrm{~h}$.

\subsection{Characterization and testing}

${ }^{29} \mathrm{Si}$ MAS solid state nuclear magnetic resonance (NMR) spectra of the hybrid materials were recorded on a Bruker DRX-300 (7.05 Tesla) spectrometer equipped with an $4 \mathrm{~mm} \mathrm{ZrO}_{2}$ rotor, operating at $4000 \mathrm{~Hz}$ and 1000 scans. The chemical shifts positions are referenced to kaolinite.

Dynamic mechanical analyzer (Q-800, TA Scientific) was employed for measuring dynamic mechanical properties of cured epoxy networks, at a fixed frequency of $10 \mathrm{~Hz}$, with a heating rate of $3^{\circ} \mathrm{C} / \mathrm{min}$. A single cantilever clamp was used for samples of size $25 \times 10 \times 2 \mathrm{~mm}^{3}$.

Thermogravimetric analysis was performed on a Q-50 thermogravimetric analyzer, TA Scientific, operating from 30 to $750^{\circ} \mathrm{C}$, under nitrogen atmosphere and a heating rate $=5^{\circ} \mathrm{C} \cdot \mathrm{min}^{-1}$.

The Small Angle X-ray Scattering (SAXS) study was performed at room temperature using synchrotron the beam line of National Synchrotron Light Laboratory (LNLS), Campinas, Brazil. This beamline is equipped with an asymmetrically cut and bent silicon (111) monochromator that yielded a monochromatic $(\lambda=1.608 \AA)$ and horizontally focused beam. A position sensitive X-ray detector and a multichannel analyzer were used to record the SAXS intensity, $I(q)$, as a function of modulus of scattering vector $q, q=(4 \pi / \lambda) \sin (\theta / 2), \theta$ being the scattering angle. Each SAXS pattern corresponds to a data collection time of $900 \mathrm{~s}$.

\section{Results and discussion}

\subsection{Condensation degree of silicon species in POSS-PMMA hybrid materials}

The hydrolysis/condensation of MPTS simultaneously to the free radical copolymerization of MPTS and MMA gives rise to a crosslinked hybrid structure as illustrated in Figure 1 [42, 43]. The structural features of the silsesquioxane moieties of the Silsesquioxane-PMMA (SSO-PMMA) hybrids prepared in this study were investigated by ${ }^{29} \mathrm{Si}$ MAS

Table 1. Composition Silsesquioxane-PMMA hybrid materials

\begin{tabular}{|c|c|c|c|c|c|c|c|c|}
\hline \multirow[b]{2}{*}{ Code } & \multicolumn{2}{|c|}{ MPTS } & \multicolumn{2}{|c|}{ MMA } & \multirow{2}{*}{$\begin{array}{c}\text { BPO } \\
{[\mathrm{mmol}]}\end{array}$} & \multicolumn{3}{|c|}{ Silsesquioxane-PMMA composition } \\
\hline & [g] & [mmol] & [g] & [mmol] & & $\begin{array}{c}\text { Siloxane } \\
{[\%]}\end{array}$ & $\begin{array}{c}\text { PMMA } \\
{[\%]}\end{array}$ & $\begin{array}{c}\text { Siloxane [\%] } \\
\text { from TGA }\end{array}$ \\
\hline PM1 & 0.4 & 1.68 & 0.00 & 0.0 & 0.05 & 44 & 56 & 38.7 \\
\hline PM2 & 0.4 & 1.68 & 0.34 & 3.4 & 0.15 & 20 & 80 & 18.4 \\
\hline PM3 & 0.4 & 1.68 & 0.68 & 6.8 & 0.25 & 14 & 86 & 13.9 \\
\hline PM4 & 0.4 & 1.68 & 1.36 & 13.6 & 0.45 & 8 & 92 & 10.2 \\
\hline
\end{tabular}




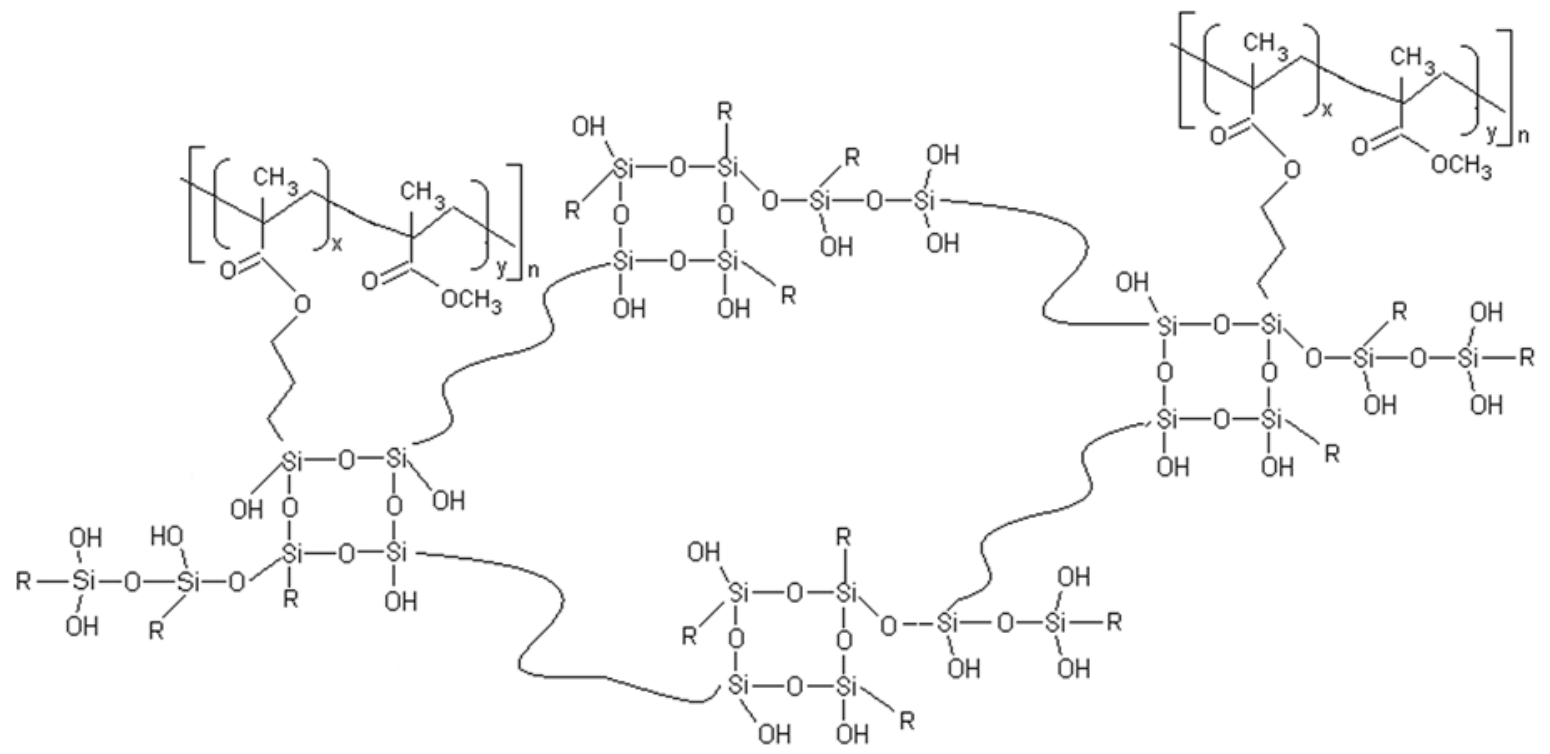

Figure 1. Proposal structure for the Silsesquioxane-PMMA hybrid material

solid state NMR spectroscopy. Figure 2 presents a typical spectrum of the hybrid compound containing 56\% of MMA (PM1).

The MMA content in the PM1 comes from the methacrylate moiety in the silane precursor, since there was no added MMA. The different species are identified according to the conventional $T^{n}$ notation, where $T$ designs the trifunctional units and $n$ corresponds to the number of neighboring silicon interconnected through the oxygen atoms of the first sphere of coordination [20, 44]. Therefore, $T^{0}$, $T^{1}$ and $T^{2}$ design the non condensed, monomeric and dimeric species, respectively, while the $T^{3}$ units refer to the completely condensed species. The ${ }^{29} \mathrm{Si}$ NMR spectra exhibits three resonance peaks at around $-50 \mathrm{ppm},-59 \mathrm{ppm}$ and $-66 \mathrm{ppm}$ associated to the presence of $T^{1}\left[\mathrm{RSi}(\mathrm{OSi})(\mathrm{OH})_{2}\right], T^{2}$ $\left[\mathrm{RSi}(\mathrm{OSi})_{2}(\mathrm{OH})\right]$ and $T^{3}\left[\mathrm{RSi}(\mathrm{OSi})_{3}\right]$, respectively. Table 2 presents the proportion of the $\mathrm{Si}$ in different environment together with the degree of condensation. The degree of condensation $(D)$ is given by Equation (1):

$D[\%]=\frac{1}{3} T^{1}+\frac{2}{3} T^{2}+\frac{3}{3} T^{3}$

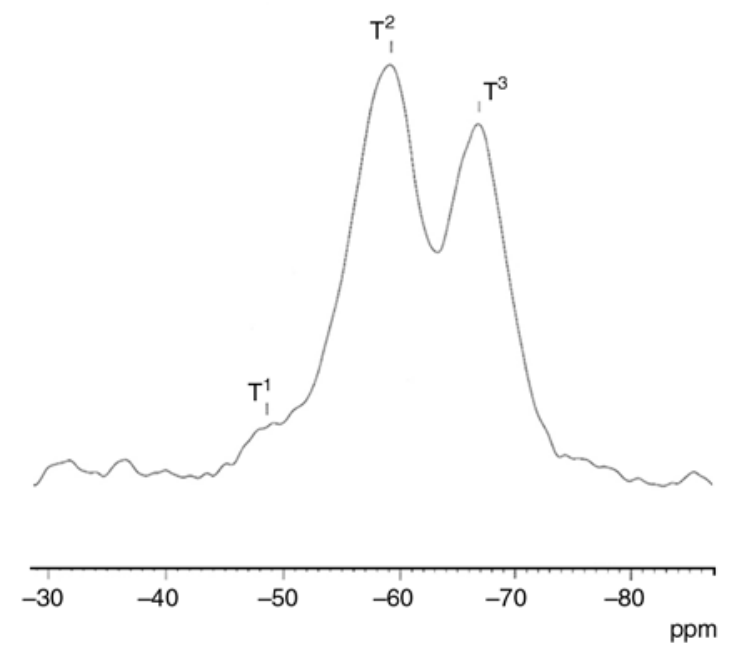

Figure 2. ${ }^{29} \mathrm{Si}$ MAS NMR spectrum of the SilsesquioxanePMMA hybrid compound containing $56 \%$ of MMA (PM1)

All hybrid materials present a high amount of silicon as dimeric species (high $T^{2}$ amount), that is, high amount of silicon bearing one hydroxyl group, indicating that the silsesquioxane prepared in this work is partially condensed. It is important to point out that the copolymerization with MMA resulted in a significant increase of the fraction of monomeric $T^{1}$ species and a pronounced decrease of the proportion of $T^{3}$ species, resulting on a

Table 2. Peak area of $T^{1}, T^{2}$ and $T^{3}$ species in Silsesquioxane-PMMA hybrid material, determined from ${ }^{29} \mathrm{Si}$ MAS solid state NMR spectroscopy

\begin{tabular}{|c|c|c|c|c|c|c|}
\hline \multirow{2}{*}{ Code } & \multicolumn{2}{|c|}{ Silsesquioxane-PMMA composition } & \multicolumn{2}{|c|}{${ }^{29}$ Si solid state NMR area [\% } & \multicolumn{2}{c|}{$\begin{array}{c}\text { Degree of } \\
\text { condensation [\%] }\end{array}$} \\
\cline { 2 - 6 } & Siloxane [\%] & PMMA [\%] & $\mathbf{T}^{\mathbf{1}}$ & $\mathbf{T}^{\mathbf{2}}$ & $\mathbf{T}^{\mathbf{3}}$ & 79 \\
\hline PM1 & 44 & 56 & 6 & 53 & 41 & 68 \\
\hline PM3 & 14 & 86 & 18 & 61 & 21 & 79 \\
\hline
\end{tabular}


decrease of the average condensation degree (Table 2). It is well known that acid $\mathrm{pH}$ conditions, as used in this study, promote hydrolysis of silicon alkoxydes but inhibit polycondensation between hydrolyzed species [45]. As a consequence, during the synthesis of Silsesquioxane-PMMA hybrids the hydrolysis of the methoxy groups of the MPTS precursor occurs firstly, but the condensation of the hydroxyl groups is slower than the free radical polymerization of MMA [46]. Therefore, the PMMA chains linked to the inorganic siloxane particles exert some hindrance towards the $-\mathrm{SiOH}$ condensation.

\subsection{Nanostructural characterization of the epoxy-based hybrid nanocomposites}

The epoxy resin was modified with SSO-PMMA hybrid compound with different compositions as described in Table 1 . The epoxy network modified with the hybrid materials were transparent indicating no discernible phase separated morphology, regardless the hybrid composition. This behavior suggests that the SSO-PMMA hybrid materials are dispersed inside the epoxy matrix in nanometric dimension.

SAXS technique is a very important tool for characterizing the nanoscopic structure of complex systems containing nanoparticles and their aggregates and/or agglomerates and provides information regarding hierarchical structures and particle size distribution $[47,48]$. This technique was employed in this work to investigate the nanostructure of the epoxy-based composites.

\subsubsection{Method of SAXS analysis}

Both asymptotic $q$-dependences, at low and high $q$, of the scattering intensity produced by a dilute set of isolated particles with no long-range correlation embedded in a homogeneous matrix having a sharp and locally flat interface, are known. In the low $q$ region, the scattering intensity produced by $N$ isolated nanoparticles is described by the Guinier law described by Equation (2):

$$
I(q)=G \exp \left(\frac{-q^{2} R_{g}^{2}}{3}\right)
$$

where $R_{g}$ is the radius of gyration of the particles and $G$ is given by Equation (3):

$$
G=N\left(\rho_{p}-\rho_{m}\right)^{2} v^{2}
$$

where $\rho_{p}$ and $\rho_{m}$ are the average electron densities of the particles and the matrix, respectively, and $v$ the average particle volume.

At high $q$ region Porod's law holds, see Equation (4):

$$
I(q)=A q^{-P}
$$

where $A=2 \pi\left(\rho_{P}-\rho_{m}\right)^{2} S, S$ being the interface area between particles and matrix and $P$ an exponent that depends of the sharpness of the interface and on the detailed geometry of the particles [48].

Dilute systems with nano-objects with different complex shapes and some polydispersivity yield a scattering intensity function $F(q)$ also obeying both Guinier and Porod laws over the low and high $q$ ranges, respectively. A semi-empirical equation proposed by Beaucage [49] that applies to the whole scattering curves and have both asymptotic behaviours described above is given by Equation (5):

$$
F(q)=G \exp \left(\frac{-q^{2} R_{g}^{2}}{3}\right)+B\left[\frac{\left(\operatorname{erf}\left(\frac{q R_{g}}{2.499}\right)\right)^{3}}{q}\right]^{P}
$$

In Equation (5), the asymptotic Guinier and Porod laws are merged. The erf function acts as a low $q$ cut-off that brings to zero the Porod low contribution at a point depending on the $R_{g}$ parameter. For globular nano-objects (neither flat disks nor thin cylinders) with smooth surface the exponent $P$ is equal to 4 while for fractal objects $1<P<3$ (in this case the exponent $P$ is the fractal dimensionality of the object).

The total intensity produced by a concentrated (not dilute) set of particles is no longer the sum of the intensity scattered by each particle. For the particular case of centro-symmetrical particles, the total intensity can be written as the product of the intensity $F(q)$ produced by a dilute system of particles (form factor) by the structure function, $S(q)$, that accounts for interference effects (Equation (6)):

$I(q)=F(q) \cdot S(q)$ 
Equation (6) applies, for example, to a set of identical spheres and can be used as a more or less good approximation for not too anisometric nanoobjects. Applying the Born-Green theory, Beaucage [49] proposed a semi-empirical and simple structure function $S(q)$ given by Equation (7):

$$
S(q)=\frac{1}{1+k \theta}
$$

where $k$ and $\theta$ are the so-called packing factor and a form function, respectively. The packing factor $k$ describes the degree of correlation that measures the number of nearest neighbor particles and it is equal to $8 V / V_{0}$ where $V$ is the average 'hard-core' volume and $V_{0}$ is the average available volume to each sphere. The form function $\theta$ depends on the average inter-particle distance $\mathrm{d}$ as follows (Equation (8)):

$$
\theta=\frac{3[\sin (q d)-q d \cos (q d)]}{(q d)^{3}}
$$

Furthermore, the total scattering intensity produced by a two-level structure can be described, under certain assumptions [49], as additive contributions from the individual levels. Nanometric domains (clusters or islands), each of them composed of a dense set of particles, embedded in a low-density matrix, give a contribution to the total intensity at very small $q$. This contribution to SAXS intensity from the second level, coarse, can also be described by Equation (2). The scattering intensity produced by domains with an average radius of gyration $R_{g_{2}}$ composed of smaller particles with an average radius of gyration $R_{g_{1}}$ is given by Equation (9) $[49,50]$ :
When the secondary domains are non spatially correlated, we have $S_{2}(q)=1$. In some cases, due to the experimental limitation imposed by the minimum accessible $q$-value, only the asymptotic Porod range of the secondary coarse level can be practically observed.

\subsubsection{SAXS results}

Figure 3 shows the SAXS patterns in log-log plot for the SSO-PMMA/epoxy composites as a function of the SSO-PMMA composition, as described in Table 1. All spectra exhibit a large broad band with a maximum located around $0.44 \AA^{-1}$ attributed to local fluctuations density of epoxy resin [51]. Moreover, it is possible to identify two other scattering regions: a decreasing linear regime at low $q$-range ( $q \leq 0.08 \AA^{-1}$ ) whose lower and upper limits change with SSO-PMMA composition and an interference peak located at higher $q$-range $(q$ around $0.09 \AA^{-1}$ ). Since the density of the siloxane phase is much higher than those of both PMMA and epoxy phases, it is possible to assume that the $\mathrm{X}$-ray scattering in these two $q$-ranges is related to the electronic density contrast between the siloxane and the polymeric phases. The presence of such two regimes has already been observed in other siloxane-polymer hybrid and composites [52] and is characteristic of the existence of a hierarchical structure consisting of two structural levels: the siloxane primary nanoparticles (first level) forming secondary aggregates distributed inside the polymer matrix (second level). Therefore, a first analysis of our SAXS results lead us to the conclusion that the primary siloxane particles concentrate in

$$
\begin{aligned}
I(q)= & {\left[G_{1} \exp \left(\frac{-q^{2} R_{g_{1}}^{2}}{3}\right)+B_{1}\left[\frac{\left(\operatorname{erf}\left(\frac{q R_{g_{1}}}{2.449}\right)\right)^{3}}{q}\right]^{P_{1}}\right] \cdot S_{1}(q)+} \\
& {\left[G_{2} \exp \left(\frac{-q^{2} R_{g_{2}}^{2}}{3}\right)+B_{2} \exp \left(\frac{-q^{2} R_{g_{1}}^{2}}{3}\right)\left[\frac{\left(\operatorname{erf}\left(\frac{q R_{g_{2}}}{2.449}\right)\right)^{3}}{q}\right]^{P_{2}}\right] \cdot S_{2}(q) }
\end{aligned}
$$




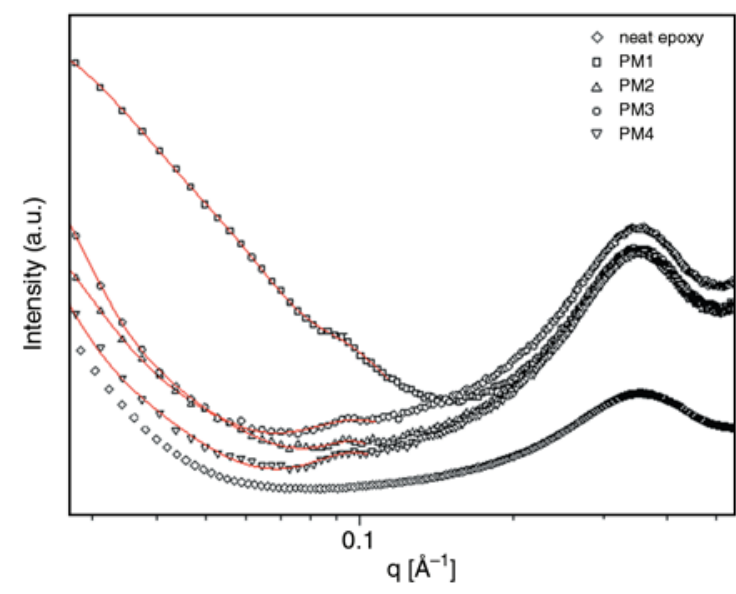

Figure 3. SAXS patterns in log-log plot for the SSOPMMA/epoxy composites as a function of the SSO-PMMA composition

relatively large nanometric domains embedded in a particles-depleted polymeric matrix.

Furthermore, for all SSO-PMMA modified epoxy systems prepared in this work, the experimental SAXS intensity curves $I(q)$ (points in Figure 3) are well fitted by the theoretical function given by Equation (8) that considers two structural levels with $S_{1}(q) \neq 1$ and $S_{2}(q) \neq 1$ (red line in Figure 3). This well fitting confirms the validity of the model of large secondary nanometric domains composed of spatially correlated quite spherical siloxane primary nanoparticles embedded in a polymeric matrix.

As discussed below, the Beaucage equation also allows obtaining quantitative information about the structural parameters of epoxy-based nanocomposites. Table 3 shows the structure parameters $G_{1}$, $R_{g_{1}}, k_{1}, d_{1}, G_{2}, R_{g_{2}}$ and $P_{2}$ determined from the best fitting procedure. The parameter $P_{1}$ has been fixed to the value 4 , assuming a smooth surface for siloxane nanoparticles. For the nanocomposite containing the PM1 hybrid (with $44 \%$ of siloxane in the hybrid (Figure 3), the siloxane particle radius was determined as around $2 \AA$, revealing the interpenetration of the SSO-PMMA with epoxy matrix at a nanometric scale. Since for this sample a tendency to Guinier plateau is observed in log-log plot, the determination of the average gyration radius $R_{g_{2}}$ of the secondary domains dispersed in the polymer matrix is possible $\left(R_{g_{2}}=90 \AA\right.$, Table 3$)$. Furthermore the value of $P_{2}$ is 1.3 , revealing that the structure of the secondary domains is a very poorly ramified (quite linear) fractal structure [53].

By increasing MMA content in the SSO-PMMA a clear decrease of the packing factor $k_{1}$ simultaneously with a shift towards lower $q$-range of the linear regime associated to the secondary large domains with a disappearance of the Guinier plateau are observed for the epoxy-based composites (Table 3 and Figure 3). The decrease of the packing factor by increasing MMA content in the hybrid copolymer is consistent with the decrease in intensity of the interference peak located around $0.09 \AA^{-1}$ as the PMMA amount in the hybrid material increases (Figure 3), both trends revealing the progressive loss of the spatial correlation between the siloxane nanoparticles located inside the secondary hybrid aggregates. This better dispersion of siloxane particles in epoxy matrix by increasing PMMA content in SSO-PMMA should be related to the lower condensation degree of the hybrid compounds containing higher MMA fraction (Table 2). As a matter of fact, the reaction between the hydroxyl groups present in the partially condensed SSO-PMMA and the anhydride used as the hardener during epoxy curing should promote chemical affinity between SSO-PMMA and epoxy, favoring siloxane nanoparticles dispersion. Another contribution should be the probable increase in PMMA chains length bonded to siloxane particles by adding more MMA in the hybrid, inhibiting siloxane particles aggregation. Note that the latter interpretation is consistent with the observed growth of the secondary aggregates size, attested by the shift of the Guinier plateau towards a $q$-range lower than the limit accessible using the setup of our experiment, as the PMMA fraction in the hybrid material increases (Figure 3). Unfortunately, the very short extension of the linear regime at low $q$ values and the absence of a trend toward a

Table 3. Structure parameters of the epoxy network modified with SSO-PMMA hybrid materials (6\%), obtained by SAXS

\begin{tabular}{|c|c|c|c|c|c|c|c|}
\hline \multicolumn{2}{|c|}{ SSO-PMMA composition [\%] } & \multirow{2}{*}{$\underset{\text { (arb.units) }}{\mathbf{G}_{2}}$} & \multirow{2}{*}{$\begin{array}{l}\mathbf{R}_{\mathbf{g}_{2}} \\
{[\AA]}\end{array}$} & \multirow{2}{*}{$\begin{array}{c}\mathbf{G}_{1} \\
\text { (arb.units) }\end{array}$} & \multirow{2}{*}{$\begin{array}{l}\mathbf{R}_{\mathbf{g}_{1}} \\
{[\AA]}\end{array}$} & \multirow{2}{*}{$\mathbf{k}_{1}$} & \multirow{2}{*}{$\begin{array}{c}\mathbf{d}_{1} \\
{[\AA]}\end{array}$} \\
\hline Siloxane & PMMA & & & & & & \\
\hline 44 & 56 & $3.7 \cdot 10^{-6}$ & 90 & $1.5 \cdot 10^{-7}$ & 1.9 & 4.4 & 54 \\
\hline 20 & 80 & - & - & $1.6 \cdot 10^{-7}$ & 1.2 & 2.6 & 53 \\
\hline 14 & 86 & - & - & $2.8 \cdot 10^{-7}$ & 1.8 & 1.3 & 53 \\
\hline 8 & 92 & - & - & $2.0 \cdot 10^{-7}$ & 1.4 & 0.8 & 54 \\
\hline
\end{tabular}


constant value for decreasing $q$ for these samples impede the determination of the average gyration radius $R_{g_{2}}$ of the secondary aggregates and also the $P_{2}$ and $G_{2}$ values. As a matter of fact in absence of Guinier plateau the values of these parameters obtained from the fit of Equation (9) have no physical meaning.

\subsection{Scanning electron microscopy}

The scanning electron micrographs of epoxy modified with PMMA and the corresponding SSOPMMA hybrid materials are illustrated in Figure 4. Epoxy network modified with PMMA presents some dispersed domain phase, whose size stayed in the range of $1-1.5 \mu \mathrm{m}$ (Figure $4 \mathrm{c}$ ). The size domains are micrometric because of the quite good compatibility between epoxy matrix and PMMA. Epoxy network modified with PM1 hybrid material presents a homogeneous morphology without discernible phase separation, indicating that all the SSO-PMMA hybrid material is dispersed inside the epoxy matrix in nanometric dimension. This behavior is in agreement with the SAXS results since the average size of the aggregates in this system was calculated to be around $9 \mathrm{~nm}$. These results evidence a better interaction between the hybrid organic-inorganic PM1 compound and the epoxy network than between PMMA and epoxy, due to low PMMA content in PM1.

As the amount of PMMA in the SSO-PMMA hybrid material increases, the phase separation at a micrometric scale is observed (Figure 4b). This phenomenon of microphase segregation of PMMA in an epoxy matrix is well-known and occurs during the cure of the epoxy resin, due to the fact that hydroxyl and epoxy groups, which promote chemical affinity between non-cured epoxy resin and PMMA, are changed into other groups during this process [54]. The microdomain sizes are even a little bit larger than that observed for epoxy/PMMA network, suggesting the presence of epoxy resin inside the domains, increasing their size. This result is consistent with the low condensation degree of PM3 determined from ${ }^{29} \mathrm{Si}$ NMR, which should induce a reaction between the hydroxyl groups present in the partially condensed SSO-PMMA and the anhydride used as the hardener. This phenomenon promotes good chemical affinity between the hybrid and epoxy, favoring epoxy penetration in the microdomains. Note that the increase of PMMA content in SSO-PMMA leads to opposite effects on microstructure (investigated by SEM) and nanostructure (investigated by SAXS) of the epoxybased composites: while larger PMMA amounts promotes microphase separation of PMMA-rich domains, they avoid nanophase segregation of siloxane-rich regions.

\subsection{Dynamic mechanical properties}

The effect of the addition of SSO-PMMA hybrid material on the dynamic mechanical properties of modified epoxy networks was evaluated in terms of storage modulus and loss factor $(\tan \delta)$. Figure 5 illustrates the dependence of these dynamic mechanical properties as a function of temperature. The glass transition temperature $\left(T_{g}\right)$ was taken as the temperature at the maximum of the tan $\delta$ peak. The curves of the neat epoxy resin and that modified with PMMA were also included for comparison.

The epoxy resin modified with pure PMMA displayed lower $T_{g}$ compared with the neat epoxy network. Since epoxy chains extremities contain acrylate groups, the number of epoxy groups available to react with anhydride is lower than in neat epoxy, leading to a less efficient curing process and conse-



a)

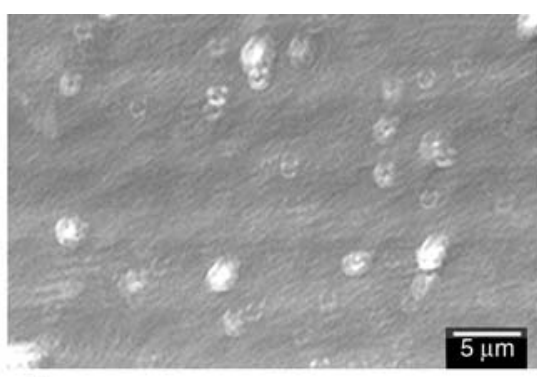

b)

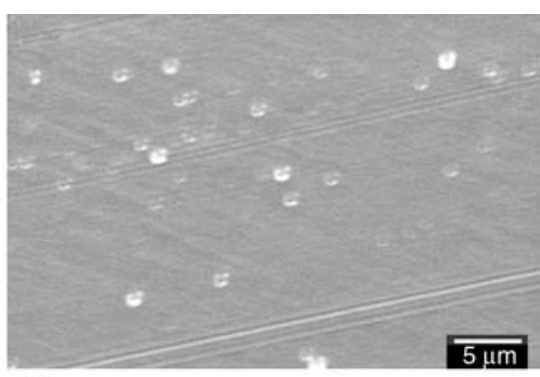

c)

Figure 4. SEM micrographs of epoxy modified with SSO-PMMA hybrid materials containing 56\% of PMMA (PM1) (a) $86 \%$ of PMMA (PM3) (b) and modified with pure PMMA (c) 

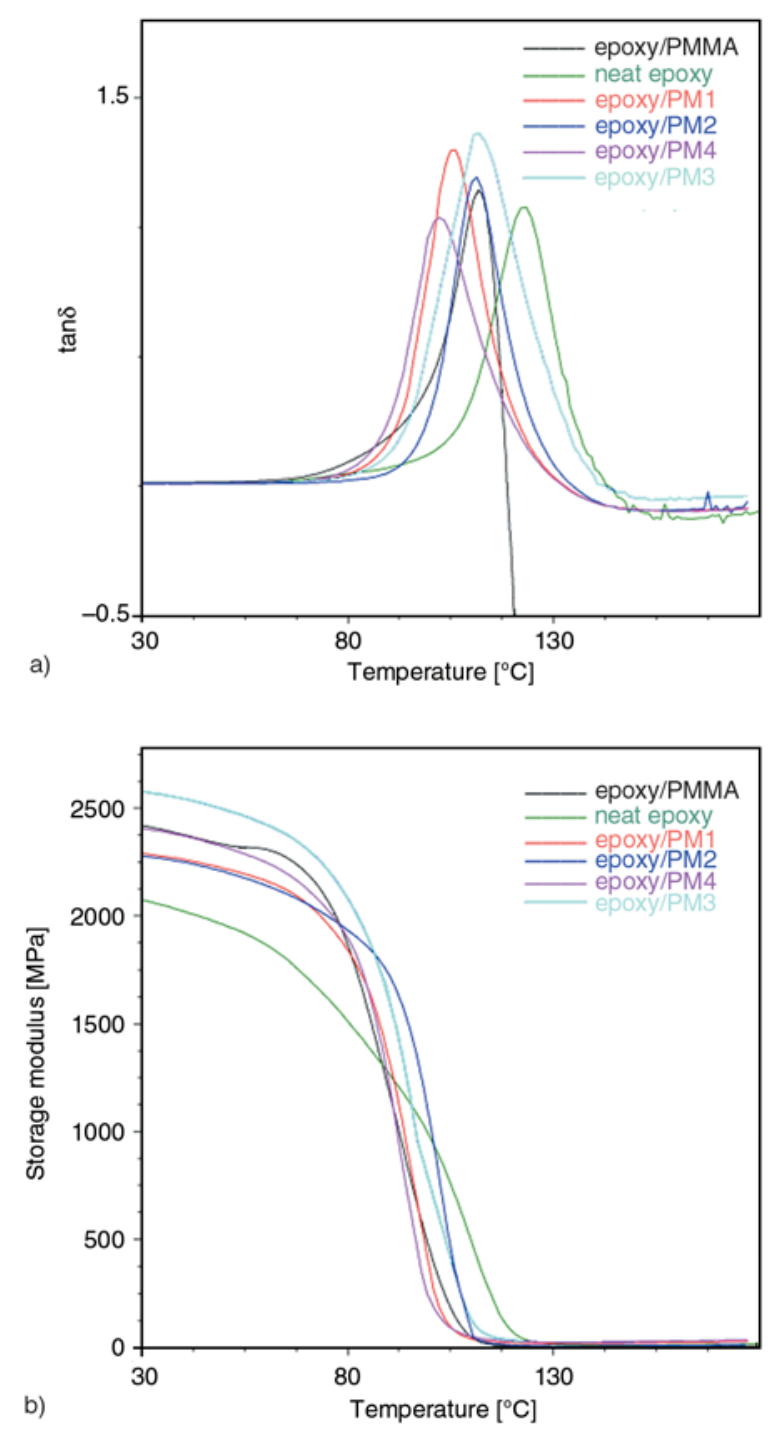

Figure 5. Dynamic mechanical properties of epoxy network modified with PMMA and SSO-PMMA hybrid materials

quently diminishing $T_{g}$. However, a plasticizing effect of PMMA because of its lower glass transition temperature than epoxy and its good compatibility with the epoxy matrix [54-56] should not be discarded.

All nanocomposites also presented lower $T_{g}$ values than the neat epoxy resin. Besides the plasticizing effect of PMMA already described, this behavior should also be due to the possible chemical reaction between silanol groups of the SSO-PMMA and anhydride. The lower amount of anhydride available for the curing process of epoxy resin induce a decrease of $T_{g}$.

A surprising result that can be observed is a significant decrease of $T_{g}$ for the nanocomposite containing PM1, which has the lowest PMMA content in the SSO-PMMA hybrid material. This phenome- non may be attributed to an increase of the free volume caused by the presence of the hybrid siloxanerich aggregates, evidenced by SAXS experiments. As a matter of fact, it has been shown that the presence of inorganic nanodomains or nanoparticles in epoxy matrix should lead to the increase of the free volume of epoxy [57].

The presence of the SSO-PMMA resulted in a significant increase of the storage modulus taken at temperature lower than the glass transition temperature, indicating a reinforcing action of the hybrid material on the epoxy matrix. The increase of PMMA content in the hybrid increased the modulus and the highest value was achieved by using the PM3 hybrid material. The epoxy network containing the PM4 hybrid material (with higher PMMA content) presented a storage modulus comparable with that modified with pure PMMA. These results are directly correlated with the hierarchical nature of the nanostructure of the composites detected by SAXS. As a matter of fact SAXS results have revealed an increase in size of the secondary hybrid aggregates (formed by the segregation of primary siloxane particles in the epoxy matrix) by increasing the fraction of PMMA in the hybrid compound. Consequently, at least up to a PMMA fraction corresponding to $\mathrm{PM} 3$, the presence of siloxane-rich secondary hybrid domains acts as reinforcing heterogeneities dispersed in epoxy resin and consequently the increase in size of these secondary aggregates leads to higher values of storage modulus. The modulus of the composite containing PM4 is lower than the one containing PM3 due to the quite absence of particles aggregation for this sample, evidenced by SAXS.

\section{Conclusions}

This work describes for the first time the modification of epoxy network by SSO-PMMA hybrid materials prepared by simultaneous hydrolysis/condensation of the silane precursor and polymerization of MMA. The addition of low amount of the SSO-PMMA hybrid material containing low amount of PMMA resulted in nanocomposites with no discernible phase separation. From SAXS experiments it was possible to suggest the presence of hierarchical structure characterized by organically modified siloxane nanoparticles forming larger hybrid nanoaggregates uniformly dispersed 
inside the epoxy matrix. The spatial correlation between the siloxane nanoparticles inside the aggregates were lost as the amount of PMMA in the hybrid material increased, indicating an increased ability of epoxy chains in diffusing through the hybrid aggregates as a consequence of the good affinity between the hybrid material and the epoxy matrix promoted by the reaction between the hydroxyl groups of the non condensed SSOPMMA and the anhydride which was employed as the hardener. The structural model proposed concerning the nanostructural features of epoxy/SSOPMMA composites obtained by SAXS is consistent with the thermomechanical properties of the materials investigated by DMA. The good interaction between the components resulted in a significant decrease of the glass transition temperature but an increase of the storage modulus of the epoxy network. These results are important since they show that it is possible to achieve epoxy networks with increased modulus by adding a small amount of hybrid material.

Further investigations are planned to establish the relationships between the method used in the SSOPMMA synthesis and the nanostructure and properties of the resulting epoxy-based network.

\section{Acknowledgements}

This work has been sponsored by Conselho Nacional de Desenvolvimento Científico e Tecnológico (CNPq) and Fundação de Amparo à Pesquisa do Estado do Rio de Janeiro (FAPERJ). The authors would like to thank the Brazilian Synchrotron Light Laboratory (LNLS) for the help in SAXS measurements.

\section{References}

[1] Nigam V., Setua D. K., Mathur G. N.: Failure analysis of rubber toughened epoxy resin. Journal of Applied Polymer Science, 87, 861-868 (2003).

DOI: $10.1002 / a p p .11519$

[2] Thomas R., Abraham J., Thomas S. P., Thomas S.: Influence of carboxyl-terminated (butadiene-co-acrylonitrile) loading on the mechanical and thermal properties of cured epoxy blends. Journal of Polymer Science Part B: Polymer Physics, 42, 2531-2544 (2004).

DOI: $\underline{10.1002 / p o l b .20115}$
[3] Kar S., Banthia A. K.: Use of acrylate-based liquid rubbers as toughening agents and adhesive property modifiers of epoxy resin. Journal of Applied Polymer Science, 92, 3814-3821 (2004).

DOI: 10.1002/app.20397

[4] Barcia F. L., Abrahao M. A., Soares B. G: Modification of epoxy resin by isocyanate-terminated polybutadiene. Journal of Applied Polymer Science, 83, 838849 (2002).

DOI: 10.1002/app.10079

[5] Barcia F. L., Amaral T. P., Soares B. G.: Synthesis and properties of epoxy resin modified with epoxy-terminated liquid polybutadiene. Polymer, 44, 5811-5819 (2003).

DOI: $\underline{10.1016 / S 0032-3861(03) 00537-8}$

[6] Kumar K. D., Kothandaraman B.: Modification of (DGEBA) epoxy resin with maleated depolymerized natural rubber. Express Polymer Letters, 2, 302-311 (2008).

DOI: $10.3144 /$ expresspolymlett.2008.36

[7] Li G. Z., Wang L., Toghiani H., Daulton T. L., Koyama K., Pittman C. U.: Viscoelastic and mechanical properties of epoxy/multifunctional polyhedral oligomeric silsesquioxane nanocomposites and epoxy/ ladderlike polyphenylsilsesquioxane blends. Macromolecules, 34, 8686-8693 (2001).

DOI: $\underline{10.1021 / \mathrm{ma} 011117 \mathrm{q}}$

[8] Zhou Y. X., Wu P. X., Cheng Z-Y., Ingram J., Jeelani S.: Improvement in electrical, thermal and mechanical properties of epoxy by filling carbon nanotube. Express Polymer Letters, 2, 40-48 (2008). DOI: $10.3144 /$ expresspolymlett.2008.6

[9] Crosby A. J., Lee J-Y.: Polymer nanocomposites: The 'nano' effect on mechanical properties. Polymer Reviews, 47, 217-229 (2007).

DOI: $\underline{10.1080 / 15583720701271278}$

[10] Pielichowski K., Njuguna J., Janowski B., Pielichowski J.: Polyhedral oligomeric silsesquioxanes (POSS)-containing nanohybrid polymers. Advances in Polymer Science, 201, 225-296 (2006). DOI: $\underline{10.1007 / 12 \quad 077}$

[11] Zhang J., Xu R., Yu D.: A novel poly-benzoxazinyl functionalized polyhedral oligomeric silsesquioxane and its nanocomposite with polybenzoxazine. European Polymer Journal, 43, 743-752 (2007). DOI: $\underline{10.1016 / j . e u r p o l y m j .2006 .11 .012}$

[12] Ikeda M., Saito H.: Improvement of polymer performance by cubic-oligosilsesquioxane. Reactive and Functional Polymers, 67, 1148-1156 (2007). DOI: 10.1016/j.reactfunctpolym.2007.08.008

[13] Baney R. H., Itoh M., Sakakibara A., Suzuki T.: Silsexquioxanes. Chemical Reviews, 95, 1409-1430 (1995). DOI: $10.1021 / \mathrm{cr} 00037 \mathrm{a} 012$ 
[14] Feher F. J., Budzichowski T. A.: New polyhedral oligosilsesquioxanes via the catalytic hydrogenation of aryl-containing silsesquioxanes. Journal of Organometallic Chemistry, 373, 153-163 (1989).

DOI: $10.1016 / 0022-328 X(89) 85041-7$

[15] Agaskar A. P.: Facile, high-yield synthesis of functionalized spherosilicates: Precursors of novel organolithic macromolecular materials. Inorganic Chemistry, 29, 1603 (1990). DOI: $10.1021 / \mathrm{ic} 00334 \mathrm{a} 005$

[16] Sellinger A., Laine R. M.: Silsesquioxanes as synthetic platforms. 3. Photocurable, liquid epoxides as inorganic/organic hybrid precursors. Chemistry of Materials, 8, 1592-1593 (1996). DOI: $\underline{10.1021 / \mathrm{cm} 9601493}$

[17] Mascia L., Prezzi L., Lavorgna M.: Peculiarities in the solvent absorption characteristics of epoxy-siloxane hybrids. Polymer Engineering and Science, 45, 1039_ 1048 (2005). DOI: $10.1002 /$ pen.20372

[18] Liang K., Li G., Toghiani H., Koo J. H., Pittman C. U.: Cyanate ester/polyhedral oligomeric silsesquioxane (poss) nanocomposites: Synthesis and characterization. Chemistry of Materials, 18, 301-312 (2006). DOI: $10.1021 / \mathrm{cm} 051582 \mathrm{~s}$

[19] Teo J. K. H., Teo K. C., Pan B., Xiao Y., Lu X.: Epoxy/polyhedral oligomeric silsesquioxane (POSS) hybrid networks cured with an anhydride: Cure kinetics and thermal properties. Polymer, 48, 5671-5680 (2007).

DOI: $10.1016 /$ j.polymer.2007.07.059

[20] Ochi M., Matsumura T.: Thermomechanical properties and phase structure of epoxy/silica nano-hybrid materials constructed from a linear silicone oligomer. Journal of Polymer Science Part B: Polymer Physics 43, 1631-1639 (2005). DOI: $\underline{10.1002 / \text { polb.20465 }}$

[21] Çopuroglu M., O’Brien S., Crean G. M.: Effect of preparation conditions on the thermal stability of an epoxy-functional inorganic-organic hybrid material system with phenyl side group. Polymer Degradation and Stability, 91, 3185-3190 (2006). DOI: $10.1016 /$ j.polymdegradstab.2006.07.017

[22] Lee A., Lichtenahn J. D.: Viscoelastic responses of polyhedral oligosilsesquioxane reinforced epoxy systems. Macromolecules, 31, 4970-4974 (1998).

DOI: $10.1021 / \mathrm{ma} 9800764$

[23] Jones I. K., Zhou Y. X., Jeelani S., Mabry J. M.: Effect of polyhedral-oligomeric-sil-sesquioxanes on thermal and mechanical behavior of SC-15 epoxy. Express Polymer Letters, 2, 494-501 (2008).

DOI: 10.3144/expresspolymlett.2008.59

[24] Choi J., Harcup J., Yee A. F., Zhu Q., Laine R. M.: Organic/inorganic hybrid composites from cubic silsesquioxanes. Journal of American Chemical Society, 123, 11420-11430 (2001).

DOI: 10.1021/ja0107201
[25] Matejka L., Strachota A., Plestil J., Whelan P., Steinhart M., Šlouf M.: Epoxy networks reinforced with polyhedral oligomeric silsesquioxanes (POSS). Structure and morphology. Macromolecules, 37, 94499456 (2004). DOI: $10.1021 / \mathrm{ma} 0484577$

[26] Liu Y., Zheng S., Nie K.: Epoxy nanocomposites with octa(propylglycidyl ether) polyhedral oligomeric silsesquioxane. Polymer, 46, 12016-12025 (2005). DOI: $10.1016 /$ j.polymer.2005.09.056

[27] Dias Filho N. L., Cardoso C. X., Aquino H. A.: Relationships between the curing conditions and some mechanical properties of hybrid thermosetting materials. Journal of Brazilian Chemical Society, 17, 935943 (2006).

DOI: 10.1590/S0103-50532006000500018

[28] Zhang Z., Liang G., Wang X.: The effect of POSS on the thermal properties of epoxy. Polymer Bulletin, 58, 1013-1020 (2007). DOI: $10.1007 / \mathrm{s} 00289-007-0732-6$

[29] Chang K-C., Lin C-Y., Lin H-F., Chiou S-C., Huang W-C., Yeh J-M., Yang J-C.: Thermally and mechanically enhanced epoxy resin-silica hybrid materials containing primary amine-modified silica nanoparticles. Journal of Applied Polymer Science, 108, 1629 1635 (2008). DOI: 10.1002/app.27559

[30] Lee T-M., Ma C-C. M., Hsu C-W., Wu H-L.: Effect of molecular structures and mobility on the thermal and dynamical mechanical properties of thermally cured epoxy-bridged polyorganosiloxanes. Polymer, 46, 8286-8296 (2005) DOI: 10.1016/j.polymer.2005.06.069

[31] Zhang Z., Gu A., Liang G., Ren P., Xie J., Wang X.: Thermo-oxygen degradation mechanisms of POSS/ epoxy nanocomposites. Polymer Degradation and Stability, 92, 1986-1993 (2007). DOI: $10.1016 /$ j.polymdegradstab.2007.08.004

[32] Choi J., Kim S. G., Laine R. M.: Organic/inorganic hybrid epoxy nanocomposites from aminophenylsilsesquioxanes. Macromolecules, 37, 99-109 (2004). DOI: $\underline{10.1021 / \mathrm{ma030309d}}$

[33] Fu J., Shi L., Chen Y., Yuan S., Wu J., Liang X., Zhong Q.: Epoxy nanocomposites containing mercaptopropyl polyhedral oligomeric silsesquioxane: Morphology, thermal properties and toughening mechanism. Journal of Applied Polymer Science, 109, 340349 (2008). DOI: $10.1002 / a p p .27917$

[34] Liu Y-L., Chang G-P., Wu C-S., Chiu Y-S.: Preparation and properties of high performance epoxysilsesquioxane hybrid resins prepared using a maleimide-alkoxysilane compound as a modifier. Journal of Polymer Science Part A: Polymer Chemistry, 43, 5787-5798 (2005).

DOI: $\underline{10.1002 / \text { pola. } 21057}$ 
[35] Ni Y., Zheng S.: Epoxy resin containing octamaleimidophenyl polyhedral oligomeric sil-sesquioxane. Macromolecular Chemistry and Physics, 206, 20752083 (2005).

DOI: $10.1002 / \mathrm{macp} .200500267$

[36] Chang R-C., Chiang C-L., Chiu Y-C.: Synthesis, characterization, and properties of novel organic/inorganic epoxy hybrids containing nitrogen/silicon via the solgel method. Journal of Applied Polymer Science, 106, 3290-3297 (2007).

DOI: 10.1002/app.26994

[37] Saravanamuttu K., Du X. M., Najafi S. I., Andrews M. P.: Photoinduced structural relaxation and densification in sol-gel-derived nanocomposite thin films: Implications for integrated optics device fabrication. Canadian Journal of Chemistry, Revue Canadienne de Chimie, 76, 1717-1729 (1998).

[38] Tadanaga K., Ellis B., Seddon A.: Near- and midinfrared spectroscopy of sol-gel derived ormosil films for photonics from tetramethoxysilane and trimethoxysilylpropylmethacrylate. Journal of Sol-Gel Science and Technology, 19, 687-690 (2000). DOI: 10.1023/A:1008714806606

[39] Sarmento V. H. V., Frigério M. R., Dahmouche K., Pulcinelli S. H., Santilli C. V.: Evolution of rheological properties and local structure during gelation of siloxane-polymethylmethacrylate hybrid materials. Journal of Sol-Gel Science and Technology, 37, 179184 (2006)

DOI: $10.1007 / \mathrm{s} 10971-005-6625-\mathrm{y}$

[40] Amir N., Levina A., Silverstein M. S.: Nanocomposites through copolymerization of a polyhedral oligomeric silsesquioxane and methyl methacrylate. Journal of Polymer Science Part A: Polymer Chemistry, 45, 4264-4275 (2007). DOI: $10.1002 /$ pola.22168

[41] Wei Y., Yang D., Bakthavatchalam R.: Thermal stability and hardness of new polyacrylate- $\mathrm{SiO}_{2}$ hybrid sol-gel materials. Materials Letters, 13, 261-266 (1992).

DOI: $10.1016 / 0167-577 X(92) 90228-C$

[42] Sarmento V. H. V., Dahmouche K., Santilli C. V., Pulcinelli S. H., Craievich A. F.: Small-angle X-ray scattering and nuclear magnetic resonance study of siloxane-PMMA hybrids prepared by the sol-gel process. Journal of Applied Cristallography, 36, 473-477 (2003).

DOI: $10.1107 / \mathrm{S} 0021889803000384$

[43] Cornu J. F: Synthèse et Etude de la Relation Microstructure-Propriétés de Matériaux Hybrides Organiques-Inorganiques à base TMSM et Application au Renforcement des Verres. PhD Thesis, Institut National des Sciences Appliquées (INSA), Villeurbanne, France (1993).

[44] Joseph R., Zhang Z., Ford W. T.: Structure and dynamics of a colloidal silica-poly(methacrylate) composite by ${ }^{13} \mathrm{C}$ and ${ }^{29} \mathrm{Si}$ MAS NMR spectroscopy. Macromolecules, 29, 1305-1312 (1996).

DOI: $10.1021 / \mathrm{ma} 951111 \mathrm{z}$
[45] Brinker J. C., Scherer G. W.: Sol-gel science: The physics and chemistry of sol-gel processing. Academic Press, San Diego (1989).

[46] Sarmento V. H. V.: Estrutura e Propriedades de Materiais Híbridos de Siloxano-PMMA preparados pelo processo sol-gel. PhD Thesis, Universidade Estadual Paulista (UNESP), Araraquara, Brazil (2005).

[47] Guinier A., Fournet G.: Small angle scattering of X-rays. Wiley and Sons, New York (1955).

[48] Glatter O., Kratky O.: Small-angle scattering of X-ray. Academic Press, New York (1982).

[49] Beaucage G.: Approximations leading to a unified exponential power-law approach to small-angle scattering. Journal of Applied Crystallography, 28, 717 728 (1995). DOI: $10.1107 /$ S0021889895005292

[50] Beaucage G., Ulibarri T. A., Black E. P., Schaefer D. W.: Multiple size scale structures in silica-siloxane composites studied by small-angle scattering. in 'Hybrid organic-inorganic composites' (eds.: Seddon A. B., Turner M. L.) ACS Symposium Series, Albuquerque, Vol 585, 97-111 (1995).

[51] Prado L. A. S. A., Cascione M., Wichmann M. H. G., Gojny F. H., Fiedler B., Schulte K., Goerigk G.: SAXS studies on nanocomposites based on epoxy resins reinforced with Aerosil(r) nanoparticles. Hasylab Annual Report, 945-946 (2005).

[52] Dahmouche K., Carlos L. D., Bermudez V. Z., Ferreira R. A. S., Santilli C. V., Craievich A. F.: Structural modelling of Eu3+-based siloxane-poly(oxyethylene) nanohybrids. Journal of Materials Chemistry, 11, 3249-3257 (2001). DOI: $10.1039 / \mathrm{b} 104822 \mathrm{~h}$

[53] Schaefer D. W., Keefer K. D.: Fractal geometry of silica condensation polymers. Physical Review Letters, 53, 1383-1386 (1984). DOI: 10.1103/PhysRevLett.53.1383

[54] Gomez C. M., Bucknall C. B.: Blends of poly(methyl methacrylate) with epoxy resin and an aliphatic amine hardener. Polymer, 34, 2111-2117 (1993). DOI: 10.1016/0032-3861(93)90737-U

[55] Ritzenthaler S., Girard-Reydet E., Pascault J. P.: Influence of epoxy hardener on miscibility of blends of poly(methyl methacrylate) and epoxy networks. Polymer, 41, 6375-3686 (2000).

DOI: $\underline{10.1016 / \mathrm{S} 0032-3861(99) 00817-4}$

[56] Remiro P. M., Marieta C., Riccardi C. C., Mondragon I.: Influence of curing conditions on the morrphologies of a PMMA-modified epoxy matrix. Polymer, 42, 9909-9914 (2001). DOI: $\underline{10.1016 / \mathrm{S} 0032-3861(01) 00564-\mathrm{X}}$

[57] Ni Y., Zheng S., Nie K.: Morphology and thermal properties of inorganic-organic hybrids involving epoxy resin and polyhedral oligomeric silsesquioxanes. Polymer, 45, 5557-5568 (2004). DOI: 10.1016/j.polymer.2004.06.008 